\section{Anemia em crianças de 6 a 59 meses e fatores associados no Município de Jordão, Estado do Acre, Brasil}

\author{
Anemia in children 6 to 59 months of age and \\ associated factors in Jordão, Acre State, Brazil
}

\author{
1 Universidade Federal do \\ Acre, Rio Branco, Brasil. \\ 2 Faculdade de Saúde \\ Pública, Universidade de \\ São Paulo, São Paulo, Brasil. \\ Correspondência \\ C. S. M. Oliveira \\ Universidade Federal do Acre. \\ Rodovia BR 364, Km 04 \\ Rio Branco, $A C$ \\ 69915-900, Brasil. \\ crisufac@yahoo.com.br
}

\begin{abstract}
This study investigated anemia prevalence and associated factors using a cross-sectional approach with 429 children 6 to 59 months of age in Jordão, Acre State, Brazil. Multiple Poisson regression in hierarchical models was used in the analysis. Overall anemia prevalence was $57.3 \%$ (95\%CI: 52.5\%-2.1\%). Age under 24 months [prevalence ratio - PR (95\%CI): 1.40 (1.09-1.74)], living in rural areas [PR: 1.23 (1.04-1.44)], households with 5-14 children [PR: 1.23 (1.04-1.44)], stunting [PR: 1.19 (1.01-1.39)], maternal anemia [PR: 1.18 (1.00-1.39)], and smoking during pregnancy [PR: 1.29 (1.09-1.53)] were associated with increased risk of anemia. Children of working mothers were at lower risk of anemia [PR: 0.78 (0.64-0.94)]. Anemia prevalence in childhood was found to be a serious public health problem in this municipality. Multi-sector prevention strategies should be implemented, addressing poverty and maternal-infant healthcare.
\end{abstract}

Anemia; Child Welfare; Nutritional Status; Prevalence
Cristieli Sérgio de Menezes Oliveira 1,2

Marly Augusto Cardoso 2

Thiago Santos de Araújo 1

Pascoal Torres Muniz 1

\section{Introdução}

A anemia permanece como a carência nutricional mais prevalente no mundo, afetando 1,62 bilhão de pessoas ou $24,8 \%$ da população mundial. O distúrbio acomete, principalmente, crianças $(47,4 \%)$, gestantes $(41,8 \%)$ e mulheres em idade fértil (30,2\%), sobretudo em países em desenvolvimento 1,2 , embora ocorra também, em menor escala, em países desenvolvidos ${ }^{3}$. Segundo a Organização Mundial da Saúde (OMS) ${ }^{2}$, entre os países da América Latina cujas prevalências de anemia são consideradas grave problema de saúde pública ( $>40 \%$ ) entre pré-escolares, incluemse: Brasil, Bolívia, Peru e Guiana.

No Brasil, até o ano de 2008 não existiam diferenças marcantes nas prevalências de anemia em menores de cinco anos entre as macrorregiões 4,5 , e os estudos populacionais loco-regionais nesta faixa etária indicavam valores de $40 \%$ a $50 \%$ 6,7,8. Em populações indígenas, entretanto, as prevalências de anemia alcançam proporções superiores a $60 \%$ 9,10. Os resultados da Pesquisa Nacional de Demografia e Saúde da Criança e da Mulher (PNDS/2006) apontam um cenário mais favorável para a prevalência da anemia no país, quando comparados com os achados de estudos anteriores realizados no Brasil, sobretudo na Região Norte. Segundo a PNDS/2006, a prevalência nacional de anemia para os menores de cinco anos foi de $20,9 \%$, sugerindo a Região Norte 
com a menor prevalência entre as macrorregiões (10,4\% de anêmicos nesta faixa etária) ${ }^{11}$.

É imprescindível o conhecimento sobre os fatores que influenciam no desenvolvimento da anemia. Embora a ferropenia responda como a principal causa, sobretudo, em populações pobres, são muitos os fatores associados a esse desfecho, como causas genéticas, infecções, malária e deficiência de outros micronutrientes bem como, o consumo inadequado de alimentos ricos em ferro e a alta ingestão de substâncias quelantes deste mineral 3,12,13. As precárias condições socioeconômicas e ambientais também possuem fortes vínculos com essa carência 8,14,15.

Em virtude da escassez de dados nacionais acerca da magnitude e distribuição geográfica da deficiência de ferro, a prevalência de anemia em crianças menores de cinco anos tem sido utilizada como sinônimo da prevalência de anemia ferropriva ${ }^{3}$. Mahoney Jr. 16 ressalta que a anemia não deve ser considerada a medida mais importante na avaliação de deficiências nutricionais, já que em estados de deficiência de ferro ela é a última constatação a aparecer e a primeira a resolver com a terapia.

As mudanças favoráveis dos indicadores de saúde e nutrição infantil descritas no Brasil nos últimos anos são decorrentes, dentre vários fatores, da maior cobertura das ações básicas de saúde, com ênfase na cobertura vacinal e assistência pré-natal e ao parto; melhoria no saneamento básico e da renda familiar; aumento da escolaridade materna 17,18; e estímulo constante promovido pelo setor saúde e pela mídia ao aleitamento materno ${ }^{18}$. No entanto, o impacto dessas mudanças não se deu de forma homogênea entre as regiões. Um exemplo disso é a prevalência da desnutrição infantil que, apesar da redução expressiva em todo país, ainda permanece alta na Região Norte (14,7\%) 11. Para a anemia, até a publicação da PNDS/2006 11, o que prevalecia era uma tendência de aumento mesmo em regiões socioepidemiológicas distintas, com as prevalências apresentadas sendo superiores 6,19 à encontrada nacionalmente e de suas respectivas regiões ${ }^{11}$. Para o Norte não foram encontrados estudos que documentassem essa tendência, indicando a necessidade de mais estudos nessa perspectiva, especialmente no contexto do interior da Amazônia.

Segundo o Fundo das Nações Unidas para a Infância (UNICEF) 20, o Índice de Desenvolvimento Infantil (IDI) na Região Norte é bem mais baixo $(0,56)$ que o observado nas regiões Sul $(0,75)$ e Sudeste $(0,78)$. No Estado do Acre, a grande maioria dos municípios possui estimativas muito altas de prevalência de desnutrição infantil, destacando-se o Município de Jordão com as piores estimativas de prevalência de desnutrição segundo o indicador de déficit de estatura para idade $(44,6 \%) 21$.

Portanto, dada a escassez de estudos sobre a prevalência e fatores associados a distúrbios nutricionais na Região Norte do país, sobretudo em áreas de difícil acesso, este estudo teve como objetivo analisar a prevalência de anemia e fatores associados no Município de Jordão.

\section{Metodologia}

\section{Área de estudo}

Este estudo foi realizado no Município de Jordão, localizado a $640 \mathrm{~km}$ em linha reta 22 de Rio Branco, capital acreana, fazendo fronteira com os municípios brasileiros de Marechal Thaumaturgo, Feijó, Tarauacá e com o Peru. Situado no vale do Juruá, ocupa uma área de $5.429 \mathrm{~km}^{2}$, banhada pelos rios Jordão e Tarauacá. No ano 2000 o Índice de Desenvolvimento Humano (IDH) foi de 0,475 , penúltimo lugar no ranking entre os municípios brasileiros (Programa das Nações Unidas para o Desenvolvimento. http://www. pnud.org.br/atlas/ranking/IDH-M\%2091\%20 00\%20Ranking\%20decrescente\%20 (pelos\%20 dados\%20de\%202000).htm, acessado em 25/ Jul/2008). Até 2005, o município não dispunha de esgotamento sanitário e sua população foi estimada em 4.633 habitantes (Instituto Brasileiro de Geografia e Estatística. http:/ /www.ibge. gov.br/munic2005/ver_tema.php?tema $=$ t9\&m unic $=120032 \& u f=12 \&$ nome $=$, acessado em 03/ Fev/2011), dos quais 30\% são indígenas Kaxinawá. É um município marcado pelo isolamento, por possuir acesso somente pelas vias aérea e fluvial, cujo deslocamento à cidade mais próxima, Tarauacá, dista seis dias de viagem de barco. Sua economia baseia-se principalmente no extrativismo vegetal, comércio local e funcionalismo público.

\section{Delineamento, população de estudo e coleta de dados}

Trata-se de um estudo transversal de base populacional. Os dados foram coletados em junho de 2005, época da “estiagem” na Amazônia. Nessa ocasião, foi realizado um censo populacional na área urbana do município, obtendo-se informação de 211 crianças (censo da zona urbana) de um total de 162 projetado para 2005. O valor 162 foi estimado com base na proporção $(0,1625)$ de crianças menores de cinco anos residentes na zonaurbana, multiplicado pela população demenores de cinco anos estimada para $2005(\mathrm{n}=998) 23$. 
$\mathrm{Na}$ área rural, por dificuldades logísticas de acesso às aldeias indígenas e às populações ribeirinhas, buscou-se incluir o maior número possível de crianças nas porções dos rios localizadas acima e abaixo da área urbana, obtendo-se uma amostra não aleatória de 254 crianças $(31,9 \%)$ de um total de 836, valor calculado baseando-se na multiplicação da proporção de menores de cinco anos para esta área $(0,8375)$ segundo dados do Censo Demográfico de 2000, pela população estimada para $2005(n=998) 23$. Obtivemos informações sobre todas as crianças residentes no Rio Tarauacá (tanto acima quanto abaixo da área urbana do município) e de cerca de um quarto das crianças residentes no rio Jordão, situado acima da área urbana. Foram elegíveis para o presente estudo 429 crianças de 6 a 59 meses. As que possuíam pelo menos um dos avós ou pais indígenas foram consideradas com ascendência indígena.

Caracterização das condições socioeconômicas, ambientais e de morbidades

Informações demográficas, socioeconômicas e ambientais foram obtidas por meio de um questionário estruturado aplicado aos pais ou responsáveis pelas crianças em entrevistas domiciliares. Analisaram-se variáveis demográficas, socioeconômicas, ambientais e maternas, além daquelas referentes ao acesso aos serviços de saúde, às práticas do aleitamento materno e à morbidade pregressa. As informações sobre peso ao nascer foram praticamente inexistentes devido à grande proporção de partos domiciliares. A data de nascimento das crianças era verificada, quando disponível, por meio do cartão de vacina da criança e da própria certidão de nascimento.

Para avaliação do nível socioeconômico das famílias, calculou-se um índice de riqueza baseado na posse de bens de consumo e utilidades domésticas do domicílio conforme descrito por Filmer \& Pritchett 24. O índice de riqueza foi obtido baseando-se na análise de componentes principais com a utilização do programa XLSTAT versão 7.52 (Addinsoft; http://www.xlstat.com/en/ home/). O cálculo foi realizado separadamente para cada uma das áreas. Pelo fato de a área rural não dispor de energia fornecida pelo serviço público, não foram considerados na análise os bens que dependem de energia elétrica. No caso da avaliação dos itens de consumo dos domicílios na zona rural, do primeiro componente principal, que explicou 45,9\% da variabilidade total, foram derivados pesos (entre parênteses) para cada item de consumo presente no domicílio: fogão $(0,594)$, rádio $(0,702)$, barco a remo $(0,720)$. As demais variáveis foram retiradas em virtude do baixo poder explicativo no índice.
Após a padronização dos pesos atribuídos aos itens de consumo avaliados, os maiores escores (positivos) foram associados à presença no domicílio de fogão $(1,676)$, rádio $(1,164)$ e barco a remo $(0,899)$, e os menores escores (negativos) para ausência de fogão $(-0,209)$, rádio $(-0,419)$ e barco a remo $(-0,572)$. Na zona urbana, do primeiro componente principal $(50,5 \%$ da variabilidade total) foram derivados pesos (entre parênteses) para cada item de consumo presente no domicílio: energia $(0,660)$, televisão $(0,770)$, som $(0,625)$, fogão $(0,636)$, geladeira $(806)$, telefone $(0,650)$, liquidificador $(0,722)$, bicicleta $(0,622)$, ferro $(0,742)$, jogo de estofado $(0,733)$ e antena parabólica $(0,813)$. Após a padronização dos pesos atribuídos aos itens de consumo avaliados, os maiores escores (positivos) foram associados à presença no domicílio de telefone $(1,381)$, jogo de estofado $(1,249)$, ferro de passar $(1,088)$, antena parabólica $(1,087)$, liquidificador $(1,058)$, aparelho de som $(0,976)$, e os menores escores (negativos) relacionados à ausência de energia $(-1,164)$, fogão $(-1,047)$, geladeira $(-0,881)$, televisão $(-0,820)$ e antena parabólica $(-0,606)$. Ao final, todos os escores, de cada área, foram somados, obtendo-se uma estimativa do índice de riqueza por domicílio e categorizados em terços.

\section{Avaliação antropométrica}

O peso e a estatura foram obtidos segundo técnicas preconizadas pela OMS 25 , utilizando-se o valor médio de duas medições. Para a pesagem e medida de comprimento das crianças menores de dois anos utilizou-se, respectivamente, balança portátil (Soehnle, Alemanha), com capacidade de $16 \mathrm{~kg}$ e precisão de $10 \mathrm{~g}$, e infantômetro portátil, com precisão de $1 \mathrm{~mm}$, colocado sobre uma superfície plana. Para as crianças mais velhas, utilizou-se balança portátil digital eletrônica (Tanita, modelo BF 682), com capacidade de $150 \mathrm{~kg}$ e precisão de $100 \mathrm{~g}$, e estadiômetro com precisão de $0,1 \mathrm{~cm}$. O índice utilizado, no presente estudo, para a avaliação do estado nutricional das crianças foi altura/idade (A/I) sendo transformado em escore $z$, tendo como referência as curvas de crescimento da OMS, com a utilização do programa Anthro 2005 (http://www.who.int/childgro wth/software/en/) 26. Crianças com índice A/I inferior ao ponto de corte de -2 escore $Z$ foram consideradas com déficit de A/I.

\section{Diagnóstico de anemia}

A dosagem de hemoglobina $(\mathrm{Hb})$ foi realizada em amostra sanguínea obtida por punctura digital, utilizando-se fotômetro portátil (Hemocue). Para definição de anemia adotou-se ponto de corte 
estabelecido pela OMS, $\mathrm{Hb}<11,0 \mathrm{~g} / \mathrm{dL}$, para as crianças de seis meses a cinco anos de idade ${ }^{3}$. A anemia foi considerada leve para valores de $\mathrm{Hb}$ entre 90 e $110 \mathrm{~g} / \mathrm{L}$; moderada quando $\mathrm{Hb}$ esteve entre 70 e $90 \mathrm{~g} / \mathrm{L}$; e grave para valores menores que $70 \mathrm{~g} / \mathrm{L}$, segundo recomendação de Gleason \& Scrimschaw 27.

\section{Processamento, análise dos dados}

e aspectos éticos

Os questionários foram duplamente digitados no programa Epi Info 6.04 (Centers for Disease Control and Prevention, Atlanta, Estados Unidos). Em seguida, as digitações foram comparadas, procedendo-se a verificação de sua consistência. Para verificar a associação entre as variáveis utilizou-se o teste do qui-quadrado de Pearson, considerando o nível de significância $\mathrm{p}<0,05$.

A razão de prevalência (RP) foi calculada por modelos múltiplos de regressão de Poisson, com estimativa de erro robusto e seleção hierárquica das variáveis independentes, com o auxílio do programa Stata version 10 (Stata Corp., College Station, Estados Unidos). Foi adotado o modelo conceitual adaptado de Silva et al. 14 , sendo incluídas todas as variáveis independentes que na análise de associação bruta apresentaram valor de $\mathrm{p}<0,20$. Iniciou-se a análise do modelo múltiplo do bloco distal para o proximal. As variáveis sexo, idade e ascendência indígena foram introduzidas no primeiro bloco e permaneceram nas etapas seguintes. As variáveis com pelo menos uma categoria com valor de $\mathrm{p}<0,05$ no teste de Wald foram selecionadas como fatores associados à anemia, permanecendo nas análises dos blocos subsequentes. As demais variáveis ( $\mathrm{p}>$ $0,05)$ foram retiradas e novamente testadas uma a uma no modelo quanto a alterações em mais de $10 \%$ na magnitude das razões de prevalência das variáveis pertencentes ao bloco 28 . Detectadas essas alterações, a variável era reinserida e permanecia no modelo dos blocos subsequentes.

Após os devidos esclarecimentos, os pais ou responsáveis assinaram voluntariamente o Termo de Consentimento Livre e Esclarecido, permitindo a participação de seus filhos na pesquisa, sendo-lhes assegurada a confidencialidade das informações prestadas. O presente estudo foi aprovado pelos Comitês de Ética em Pesquisa da Fundação Hospital Estadual do Acre (FUNDHACRE) e da Faculdade de Saúde Pública da Universidade de São Paulo. As crianças anêmicas receberam tratamento pela equipe médica do projeto.

\section{Resultados}

A prevalência geral de anemia foi de $57,3 \%$ (IC95\%: 52,5\%-62,1\%). Considerando-se a população de crianças com ascendência indígena residente na área urbana $(n=56)$, a prevalência de anemia foi de $37,5 \%$. A situação se agravou para aqueles residentes na zona rural $(n=115)$, onde 64,3\% das crianças com ascendência indígena eram anêmicas. A Figura 1 apresenta as prevalências de anemia em crianças de 6 a 59 meses, segundo diferentes pontos de corte para $\mathrm{Hb}$, zona de moradia e ascendência indígena. Somente um caso de anemia grave foi encontrado entre crianças residentes na área rural $(\mathrm{Hb}<70 \mathrm{~g} / \mathrm{L})$. Os valores de $\mathrm{Hb}$ variaram de 68 a 151g/L (média: 107g/L; DP: 14g/L).

Em relação às características ambientais, não havia esgotamento sanitário no município em 2005: 75\% dos dejetos eram desprezados em fossa ou vala a céu aberto, $30 \%$ das crianças consumiam água sem nenhum tipo de tratamento e cerca de $60 \%$ eram provenientes de famílias com renda inferior a 288 Reais (dados não apresentados em tabela).

Quanto à distribuição das características sociodemográficas nessa população (Tabela 1), a prevalência de anemia foi semelhante entre os sexos, predominando em crianças de 6 a 23,9 meses $\left(75 \% ; p=0,0001 ; \chi^{2}=33,1\right)$, que residiam na área rural $(62,1 \%)$, em moradias de paxiúba ou barraco $(62,9 \%)$ e com alta aglomeração intradomiciliar (64\%). Observou-se que as crianças não inscritas em algum programa social $(60,9 \%)$, pertencentes às famílias mais pobres $(62,7 \%)$ e cujas mães não trabalhavam fora $(63,1 \%)$ tiveram maior risco de apresentar anemia.

A respeito das condições de acesso ao serviço básico de saúde, observou-se que $62,6 \%$ das crianças nasceram no próprio domicílio e somente $17,5 \%$ referiram acompanhamento regular em serviços de atenção básica (predominantemente em caso de adoecimento). Somente $17,8 \%$ das crianças estudadas apresentavam esquema vacinal em dia (dados não apresentados em tabela).

A Tabela 2 apresenta a prevalência de anemia e as razões de prevalência brutas segundo variáveis maternas. Houve maior prevalência de anemia entre crianças cujas mães eram anêmicas $(67,3 \%)$, adolescentes $(73,1 \%)$, ou que fumaram durante a gravidez (66,5\%). A utilização de remédio para verme nos últimos doze meses mostrou diferença estatisticamente significante na prevalência de anemia, com risco $23 \%$ maior para as crianças que não fizeram uso do medicamento. Dezessete por cento das mães não sabiam ler ou 
Prevalências de anemia segundo gravidade em crianças de 6 a 59 meses, de acordo com a zona de moradia e ascendência indígena. Jordão, Acre, Brasil, 2005 ( $n=429$ ).

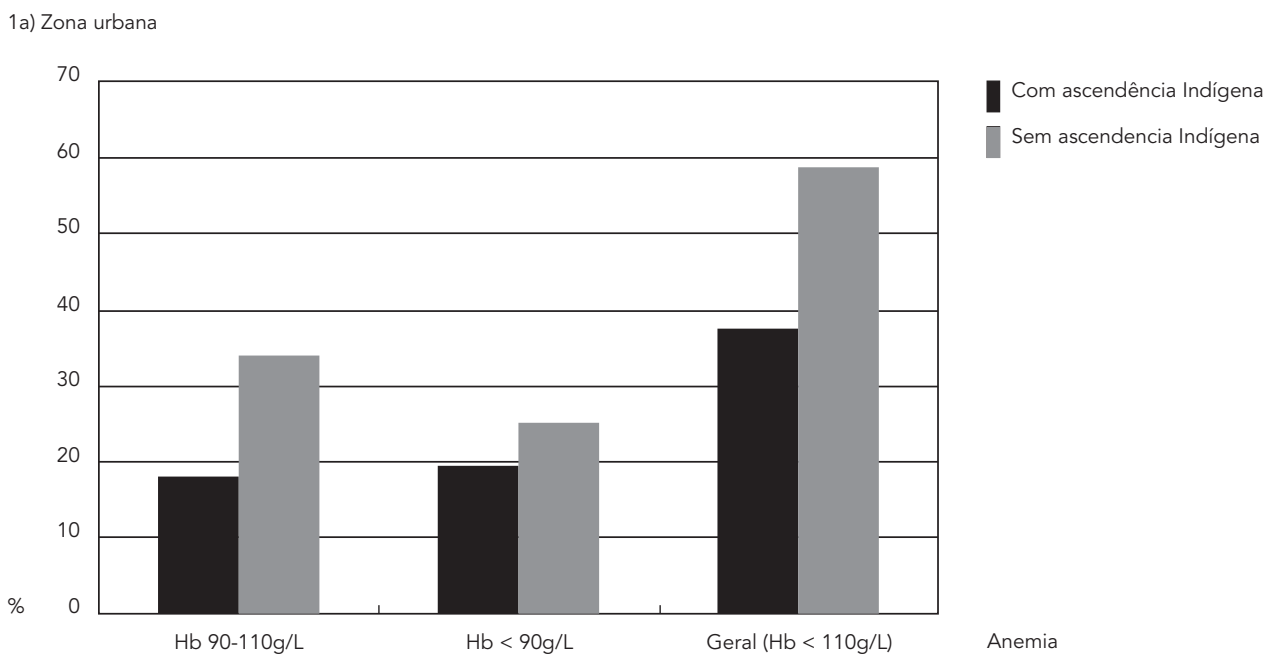

1b) Zona rural

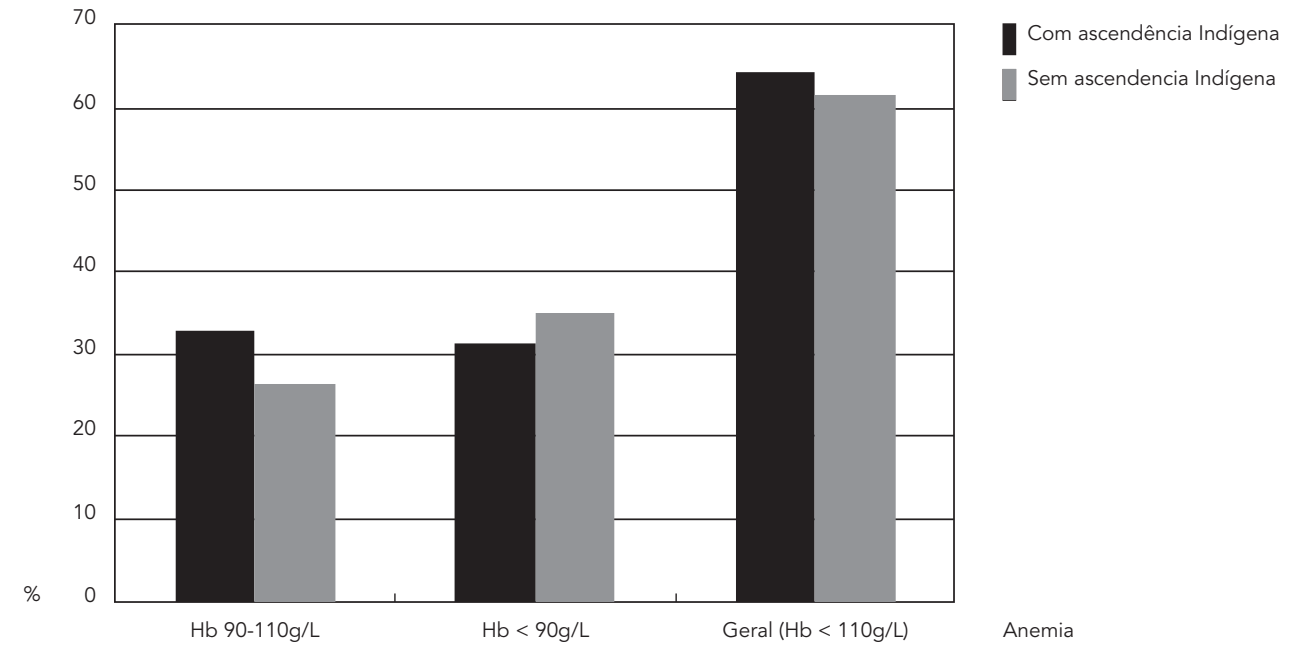

nunca frequentaram a escola (dados não apresentados em tabela).

A Tabela 3 apresenta as razões de prevalências com associação positiva e estatisticamente significante entre anemia e tempo de aleitamento materno total (AMT) inferior a 120 dias (RP: 1,54) comparado à categoria de maior tempo de AMT; naquelas vivendo em famílias que tinham disponibilidade de leite de vaca para consumo (28\% maior risco de apresentar anemia), para as crianças que tiveram diarréia recente (RP: 1,19) e entre as que apresentaram pneumonia no último ano (19\% maior risco de ter anemia). O efeito protetor do aleitamento materno exclusivo superior a 120 dias em relação à categoria de menor valor foi de 27\% (IC95\%: 0,55-1,00; $\mathrm{p}=0,049$ ), embora limítrofe.

$\mathrm{O}$ aleitamento materno foi iniciado para a maioria das crianças $(92,2 \%)$, mas a prática de aleitamento materno exclusivo foi precocemente 
Prevalência de anemia e razões de prevalência brutas, conforme características demográficas e socioeconômicas de crianças de 6 a 59 meses. Jordão, Acre, Brasil, 2005 ( $n=429)$.

\begin{tabular}{|c|c|c|c|c|}
\hline Variável & $n$ * & $\begin{array}{l}\text { Prevalência de } \\
\text { anemia (\%) ** }\end{array}$ & $\mathrm{RP}_{\text {bruta }} * \star \star$ & IC95\% \\
\hline \multicolumn{5}{|l|}{ Sexo } \\
\hline Feminino & 220 & 54,5 & 1,00 & \\
\hline Masculino & 209 & 60,3 & 1,10 & $0,93-1,30$ \\
\hline \multicolumn{5}{|l|}{ Idade (em meses) } \\
\hline $6-23,9$ & 156 & 75,0 & 1,38 & $1,09-1,74$ \\
\hline $24-47,9$ & 201 & 44,8 & 0,82 & $0,63-1,07$ \\
\hline$\geq 48$ & 72 & 54,2 & 1,00 & \\
\hline \multicolumn{5}{|l|}{ Ascendência indígena \# } \\
\hline Não & 241 & 60,2 & 1,00 & \\
\hline Sim (neto ou filho de indígena) & 171 & 55,6 & 0,92 & $0,77-1,09$ \\
\hline \multicolumn{5}{|l|}{ Zona de moradia } \\
\hline Urbana & 186 & 51,1 & 1,00 & \\
\hline Rural & 243 & 62,1 & 1,21 & $1,02-1,44$ \\
\hline \multicolumn{5}{|l|}{ Tipo do domicílio } \\
\hline Alvenaria/Madeira/Mista & 211 & 51,2 & 1,00 & \\
\hline Barraco/Paxiúba & 194 & 62,9 & 1,07 & $1,01-1,13$ \\
\hline \multicolumn{5}{|l|}{ Índice de riqueza } \\
\hline 1ㅇq quartil & 153 & 62,7 & 1,10 & $0,83-1,22$ \\
\hline 2o quartil & 50 & 40,0 & 0,64 & $0,44-0,93$ \\
\hline 3o tercil & 123 & 53,7 & 0,86 & $0,69-1,07$ \\
\hline 4o tercil (alto) & 103 & 62,1 & 1,00 & \\
\hline \multicolumn{5}{|l|}{ Número de crianças no domicílio } \\
\hline $1-5$ & 312 & 54,81 & 1,00 & \\
\hline $6-14$ & 114 & 64,04 & 1,16 & $0,98-1,38$ \\
\hline \multicolumn{5}{|c|}{ Está inscrita em algum programa social } \\
\hline Não & 299 & 60,9 & 1,00 & \\
\hline Sim & 119 & 49,6 & 0,81 & $0,66-0,99$ \\
\hline \multicolumn{5}{|l|}{ Mãe trabalha fora } \\
\hline Não & 271 & 63,1 & 1,00 & \\
\hline Sim & 147 & 48,2 & 0,76 & $0,63-0,93$ \\
\hline
\end{tabular}

* As diferenças nos valores absolutos das frequências correspondem às perdas;

** Qui-quadrado de Pearson;

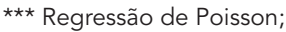

\# Foi considerado com ascendência indígena neto ou filho de indígena.

interrompida: $70 \%$ das crianças eram amamentadas exclusivamente por menos de 30 dias; houve baixa mediana de aleitamento materno exclusivo - AME (14 dias) e de AMT (360 dias). A prevalência geral do déficit de A/I foi de $37,1 \%$; entre as crianças sem ascendência indígena foi de $25,3 \%$ e nas crianças desta ascendência $52 \%$ (dados não apresentados em tabela).

A Tabela 4 apresenta as variáveis que permaneceram associadas à anemia em modelos múltiplos. Após ajuste para as variáveis do mesmo nível hierárquico e nos níveis anteriores, permaneceram associadas à presença de anemia: local de moradia, número de crianças no domicílio, mãe trabalhar fora, fumo durante a gestação, anemia materna e déficit de A/I. A variável que apresentou maior associação com o desfecho foi idade da criança, indicando que as crianças de seis a 23,9 meses mantiveram maior risco (40\%) de apresentar anemia em comparação às de 24 meses ou mais. 
Prevalência de anemia e razões de prevalência brutas, segundo características maternas e uso de remédio para verme nos últimos 12 meses em crianças de 6 a 59 meses. Jordão, Acre, Brasil, 2005 ( $n=429$ ).

\begin{tabular}{|c|c|c|c|c|}
\hline Variável & $n$ * & $\begin{array}{c}\text { Prevalência } \\
\text { de } \\
\text { anemia (\%) ** }\end{array}$ & $\mathrm{RP}_{\text {bruta }} * \star \star$ & IC95\% \\
\hline \multicolumn{5}{|l|}{ Anemia materna } \\
\hline Não & 283 & 54,1 & 1,00 & \\
\hline $\operatorname{Sim}$ & 110 & 67,3 & 1,24 & $1,05-1,47$ \\
\hline \multicolumn{5}{|c|}{ Escolaridade materna (anos) } \\
\hline Sem escolaridade & 73 & 57,5 & 0,93 & $0,77-1,11$ \\
\hline $1-4$ & 191 & 56,0 & 0,95 & $0,75-1,21$ \\
\hline$\geq 5$ & 153 & 60,1 & 1,00 & \\
\hline \multicolumn{5}{|l|}{ Idade da mãe (anos) } \\
\hline $15-19$ & 52 & 73,1 & 1,22 & $0,96-1,55$ \\
\hline $20-30$ & 204 & 51,5 & 0,86 & $0,69-1,07$ \\
\hline $31-46$ & 89 & 59,6 & 1,00 & \\
\hline \multicolumn{5}{|l|}{ Realização de pré-natal } \\
\hline Não & 235 & 58,3 & 1,16 & $0,83-1,61$ \\
\hline Sim, $<3$ consultas & 87 & 58,6 & 1,17 & $0,82-1,67$ \\
\hline Sim, 3-10 consultas & 40 & 50,0 & 1,00 & \\
\hline \multicolumn{5}{|c|}{ Fumou durante a gravidez } \\
\hline Não fuma & 210 & 50,0 & 1,00 & \\
\hline Fumou & 161 & 66,5 & 1,32 & $1,11-1,58$ \\
\hline \multicolumn{5}{|c|}{ últimos seis meses } \\
\hline Não & 187 & 64,7 & 1,23 & $1,04-1,45$ \\
\hline Sim & 227 & 52,4 & 1,00 & \\
\hline
\end{tabular}

* As diferenças nos valores absolutos das frequências correspondem às perdas;

** Qui-quadrado de Pearson;

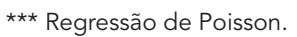

\section{Discussão}

A elevada prevalência de anemia encontrada no presente estudo $(57,3 \%)$ ressalta a magnitude do problema e a precariedade das condições nutricionais entre as crianças que vivem nessa região da Amazônia, classificando-a segundo os critérios internacionais da OMS 2 como um grave problema de saúde pública. Estudos populacionais realizados com pré-escolares em outros municípios brasileiros, como São Paulo (46,9\%) 6; Acrelândia e Assis Brasil, no Acre (30,6\%) 29; Pelotas $(30,2 \%) 30$, no Rio Grande do Sul; e no Estado de Pernambuco 15 (40,6\%), relataram valores inferiores aos encontrados neste estudo. Por sua vez, a prevalência de anemia encontrada em Jordão foi semelhante à observada nas crianças da Indonésia $(56,1 \%) 31$ e inferior à registrada para crianças indígenas brasileiras 9,10.
Embora não haja pretensão deste estudo em determinar a etiologia da carência na população estudada, acreditamos que o principal causador da anemia nessa população seja a deficiência de ferro, baseando-se nas estimativas de prevalência de anemia ferropriva descritas em estudos anteriores conduzidos na região Amazônica 32,33, bem como nas práticas inadequadas de aleitamento materno observadas. Para os especialistas, a introdução precoce do leite de vaca tem sido considerada desvantajosa para a nutrição da criança, pois além de diminuir a duração do aleitamento materno 34 , pode interferir na $a b-$ sorção de nutrientes existentes no leite humano, como o ferro 35 .

Um resultado particularmente relevante é a prevalência de anemia $20 \%$ mais baixa entre as crianças com ascendência indígena residentes na área urbana, quando comparadas às crianças 
Prevalência de anemia e razões de prevalência brutas, segundo práticas de aleitamento materno, produção familiar, morbidades referidas e déficit estatural de crianças de 6 a 59 meses. Jordão, Acre, Brasil, 2005 ( $n=429$ ).

\begin{tabular}{|c|c|c|c|c|}
\hline Variável & $n$ * & $\begin{array}{c}\text { Prevalência } \\
\text { de } \\
\text { anemia }(\%) \text { ** }\end{array}$ & $\mathrm{RP}_{\text {bruta }} * \star \star$ & IC95\% \\
\hline \multicolumn{5}{|c|}{ Aleitamento materno exclusivo (dias) } \\
\hline$<120$ & 336 & 60,4 & 0,73 & $0,53-1,00$ \\
\hline$>120$ & 54 & 44,4 & 1,00 & \\
\hline \multicolumn{5}{|c|}{ Tempo de aleitamento total (dias) } \\
\hline $0-120$ & 86 & 53,5 & 1,54 & $1,17-2,01$ \\
\hline $121-360$ & 135 & 61,5 & 1,36 & $1,04-1,77$ \\
\hline $361-760$ & 82 & 69,5 & 1,18 & $0,87-1,60$ \\
\hline$>760$ & 91 & 45,1 & 1,00 & \\
\hline \multicolumn{5}{|c|}{ Introdução de leite de vaca (dias) } \\
\hline $0-14$ & 119 & 52,94 & 1,07 & $0,79-1,46$ \\
\hline $15-30$ & 107 & 66,36 & 1,35 & $1,01-1,80$ \\
\hline $31-179$ & 91 & 58,24 & 1,18 & $0,87-1,60$ \\
\hline$>180$ & 63 & 49,21 & 1,00 & \\
\hline \multicolumn{5}{|c|}{ Disponibilidade de leite de vaca pela família } \\
\hline $\operatorname{Sim}$ & 145 & 66,9 & 1,28 & $1,09-1,51$ \\
\hline Não & 279 & 52,0 & 1,00 & \\
\hline \multicolumn{5}{|c|}{ Disponibilidade de frutas pela família } \\
\hline $\operatorname{Sim}$ & 150 & 52,0 & 0,87 & $0,72-1,04$ \\
\hline Não & 274 & 59,8 & 1,00 & \\
\hline \multicolumn{5}{|c|}{ Diarréia nos últimos 15 dias } \\
\hline Não & 227 & 53,3 & 1,00 & \\
\hline Sim & 187 & 63,6 & 1,19 & $1,01-1,40$ \\
\hline \multicolumn{5}{|c|}{ Pneumonia no último ano } \\
\hline Não & 330 & 56,1 & 1,00 & \\
\hline Sim & 73 & 67,1 & 1,19 & $0,99-1,44$ \\
\hline \multicolumn{5}{|c|}{ Déficit de A/I (-2 escore $\mathrm{z})$} \\
\hline Sim & 159 & 62,3 & 1,14 & $0,97-1,34$ \\
\hline Não & 270 & 54,4 & 1,00 & \\
\hline
\end{tabular}

* As diferenças nos valores absolutos das frequências correspondem às perdas;

** Qui-quadrado de Pearson;

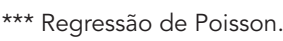

sem ascendência indígena desta área, bem como, aos grupos descritos que residem na área rural (Figura 1). Os responsáveis pelas crianças com ascendência indígena residentes na área urbana relataram maior acesso a serviços de saúde e a medicamentos, como sulfato ferroso, polivitamínicos e anti-helmínticos, amplamente distribuídos no Pólo Indígena de Saúde, dentre outras ações. Já as famílias das crianças sem ascendência indígena referiram utilizar os serviços da Unidade Mista de Saúde somente em situação de adoecimento ou de urgências. Em virtude do baixo volume das águas dos rios, situação viven- ciada em vários meses do ano, a equipe do pólo de saúde indígena fica impossibilitada de fazer viagens às aldeias, permanecendo tempo considerável na área urbana do município, o que pode explicar em parte a maior utilização deste serviço pela população com ascendência indígena residente na área urbana. Cabe ressaltar que até o ano de 2005 o município não havia implantado o Programa Saúde da Família.

Em nosso estudo, a maior prevalência de anemia entre as crianças residentes na área rural reforça o exposto em uma pesquisa realizada em Pernambuco, que evidenciou prevalência de 
Razões de prevalência após ajuste múltiplo para fatores associados à anemia em crianças de 6 a 59 meses. Jordão, Acre, Brasil, $2005(n=429)$.

\begin{tabular}{|c|c|c|c|}
\hline Variável & $\mathrm{RP}_{\text {ajustada }}$ * & IC95\% & Valor de $\mathrm{p}$ \\
\hline \multicolumn{4}{|l|}{ Sexo } \\
\hline Feminino & 1,00 & & \\
\hline Masculino & 1,13 & $0,96-1,32$ & 0,118 \\
\hline \multicolumn{4}{|l|}{ Faixa etária (meses) } \\
\hline$\geq 48$ & 1,00 & & \\
\hline $24-47,9$ & 0,83 & $0,64-1,07$ & 0,158 \\
\hline $6-23,9$ & 1,40 & $1,09-1,74$ & 0,005 \\
\hline \multicolumn{4}{|l|}{ Ascendência indígena } \\
\hline Não é filho ou neto de indígena & 1,00 & & \\
\hline Filho ou neto de indígena & 0,93 & $0,79-1,09$ & 0,389 \\
\hline \multicolumn{4}{|l|}{ Zona de moradia } \\
\hline Urbana & 1,00 & & \\
\hline Rural & 1,23 & $1,05-1,45$ & 0,013 \\
\hline \multicolumn{4}{|l|}{ Número de crianças no domicílio } \\
\hline $1-5$ & 1,00 & & \\
\hline $5-14$ & 1,23 & $1,04-1,44$ & 0,014 \\
\hline \multicolumn{4}{|l|}{ Mãe trabalha fora } \\
\hline Não & 1,00 & & \\
\hline $\operatorname{Sim}$ & 0,78 & $0,64-0,94$ & 0,011 \\
\hline \multicolumn{4}{|l|}{ Fumo durante a gravidez } \\
\hline Não & 1,00 & & \\
\hline Sim & 1,29 & $1,09-1,53$ & 0,003 \\
\hline \multicolumn{4}{|l|}{ Anemia materna } \\
\hline Não & 1,00 & & \\
\hline Sim & 1,18 & $1,00-1,39$ & 0,048 \\
\hline \multicolumn{4}{|l|}{ Déficit de altura para idade } \\
\hline Não & 1,00 & & \\
\hline Sim & 1,19 & $1,01-1,39$ & 0,033 \\
\hline
\end{tabular}

* Ajustadas pelas outras variáveis componentes do modelo.

anemia $43 \%$ maior nas crianças residentes nesta área 7 . $\mathrm{O}$ ambiente rural dificulta o acesso aos serviços de saúde e educação, bem como a aquisição de alimentos de melhor valor nutricional e favorece a introdução precoce de alimentos 5 , apontando para a conjuntura de vulnerabilidade que potencializa a chance das crianças se tornarem anêmicas.

No tocante à influência da aglomeração na ocorrência da anemia, observou-se, em Jordão, que aquelas residentes em domicílios com mais de cinco crianças apresentaram maior prevalência de anemia, corroborando os achados de estudo em Criciúma, onde crianças em situação de maior aglomeração apresentaram risco quatro vezes maior de ter anemia 36, o que também é assinalado por Matta et al. 37 no Rio de Janeiro e por Santos et al. 38 em Pelotas.
Neste estudo, não houve associação entre a presença de anemia e os níveis de escolaridade da mãe, diferenciando-se de alguns trabalhos 5,6,31 e corroborando outros 36,39 que não constataram esta associação. Deve-se considerar o contexto de baixa escolaridade (média de quatro anos) e a consequente homogeneidade dos grupos estudados no julgamento desses contrastes epidemiológicos. Por outro lado, houve associação entre a variável "mãe trabalhar fora” e anemia, sugerindo que melhores condições sociais, nessa população, se sobrepõem à melhor capacidade materna de cuidado. A participação da mulher no orçamento familiar indica melhoria no status socioeconômico da família, refletindo no poder de compra e obtenção de alimentos, embora no contexto de regiões mais ricas, como o Sudeste brasileiro 39 , o trabalho materno tenha sido regis- 
trado como fator contribuinte para o estado anêmico da criança, uma vez que ela é submetida aos cuidados de pessoas menos qualificadas.

A variável faixa etária se associou de forma estatisticamente significante com a presença de anemia. De fato, os menores de dois anos são os mais suscetíveis, conforme já descrito em estudos nacionais 5,6,7,36 e internacionais 31,40 . Essa vulnerabilidade advém, dentre outras causas, do rápido crescimento e, sobretudo, devido às práticas inadequadas de aleitamento e alimentação complementar 2,3,6,40.

A baixa mediana de AME (14 dias), com a introdução extremamente precoce do leite de vaca pode ter contribuído para a elevada prevalência de anemia. A inexistência de associação entre as práticas de aleitamento materno e anemia neste estudo concorda com os resultados encontrados em Porto Alegre 14, no qual ressaltam que o delineamento transversal não é o mais adequado para estudar esta associação por se tratar de um estudo retrospectivo em relação à prática da amamentação. Destaca ainda que, a ação protetora do aleitamento é mais evidente nos primeiros seis meses de vida, grupo normalmente não incluído em estudos sobre anemia por não apresentar valores de corte precisos para classificação de anemia e por ser, em tese, considerado protegido deste problema em virtude da reserva de ferro e do aleitamento materno.

Uma variável importante que apresentou associação com anemia foi o fumo durante a gravidez. O hábito de fumar pode provocar deficiência na absorção da vitamina B12, que está associado ao parto prematuro e à redução na eritropoiese, levando à anemia e prejuízos no crescimento fetal 41. Além disso, presume-se que na ausência da variável peso ao nascer, o tabagismo no período gestacional poderia ser considerado uma proxy do baixo peso ao nascer.

A anemia presente em $28 \%$ das mães deste estudo foi positivamente associada ao estado anêmico das crianças, sendo superior aos achados registrados em São Leopoldo, Rio Grande do Sul $(19,2 \%) 42$ e no Estado de Pernambuco $(21,6 \%) 5$. Segundo a OMS 2, 47,5\% das mulheres africanas não grávidas são classificadas como anêmicas, enquanto no sul da Ásia estas proporções estão em torno de $45,7 \%$. A associação observada no presente estudo pode refletir uma baixa cobertura dos serviços de pré-natal, bem como a semelhança existente entre o padrão alimentar de mães e filhos 29, sugerindo uma condição carencial compartilhada pelos grupos biológicos mais vulneráveis da família. Ao contrário do exposto pela PNDS/2006 11, em nossa investigação a situação de anemia entre as crianças menores de cinco anos foi duas vezes superior àquela regis- trada para as mães, o que distingue a dimensão epidemiológica do problema para cada grupo. Mesmo as mães com níveis normais de $\mathrm{Hb}$ apresentaram filhos com magnitude importante de anemia, e entre as crianças cujas mães eram anêmicas esta prevalência foi ainda maior, prevalecendo o pressuposto de que mães anêmicas têm maior probabilidade de terem filhos anêmicos, achados muito semelhantes aos já descritos por El-Saved et al. 43 no Egito. Em Pernambuco, Silva et al. 5 observaram que mães com anemia têm maior chance de ter filhos com esta carência, e que a partir dos três anos de idade estas diferenças praticamente desaparecem. Em 2006, Miglioli et al. ${ }^{44}$ confirmaram esse comportamento num segundo estudo conduzido em Pernambuco. No presente estudo, a presença de déficit de A/I foi significantemente associada ao risco para anemia. Resultados equivalentes provêm de estudo realizado no Rio de Janeiro 37 , com crianças menores de cinco anos frequentadoras de creches, os quais identificaram que crianças anêmicas apresentaram menores médias de escore $\mathrm{z}$ e maior ocorrência de desnutrição. Na Indonésia, uma pesquisa com 85.229 crianças de 6 a 59 meses observou que as que tinham déficit de A/I apresentavam maior prevalência de anemia 31 .

Há algumas limitações deste estudo que devem ser consideradas na interpretação de nossos achados. Por dificuldades logísticas não foi possível utilizar uma amostra aleatória na zona rural, o que pode comprometer a extrapolação das estimativas para esta área do município. Em virtude do tamanho amostral, a elaboração de análises separadas para as zonas urbana e rural fica comprometida em decorrência do número reduzido de indivíduos em cada categoria. Além disso, a introdução da variável zona de moradia na elaboração do modelo de identificação de fatores associados promove seu ajuste. A ausência de informações acuradas relativas às condições de gestação e parto, tais como peso ao nascer e a idade gestacional, impossibilitaram uma análise mais abrangente dos determinantes da anemia na região. Com relação à avaliação do estado nutricional, devido a dificuldades de transporte e limitações financeiras do projeto não foi possível obter indicadores hematimétricos e bioquímicos necessários ao diagnóstico e estimativa da magnitude da deficiência de ferro. Infelizmente, não se dispõe de dados sobre o consumo alimentar, necessários para estimativas de ferro biodisponível na dieta; em adição, não foi incluída a variável raça/cor que tem potencial mais preciso na caracterização étnica da população.

Apesar das limitações referidas, o levantamento dessas informações in loco constitui iniciativa ímpar para o estabelecimento de um 
diagnóstico pioneiro sobre saúde e nutrição dessas crianças, que sobrevivem num meio onde os desafios relativos à organização de serviços de infraestrutura básica - como fomentação da agricultura de subsistência local, geração de renda e qualificação dos serviços de saúde - ainda precisam ser superados.

Os resultados aqui apresentados confirmam a determinação social do problema, visto que os fatores sociais foram identificados como importantes preditores da anemia. O enfrentamento da anemia nesse município requer, dentre outras medidas, maior cobertura das ações básicas de saúde com ênfase na assistência pré-natal e de puericultura. Apesar de se tratar de um município pequeno, a realidade observada é compartilhada por vários outros municípios do interior da Amazônia que ainda apresentam grande parcela das pessoas residindo em suas áreas rurais, onde as dificuldades de acesso a serviços essenciais como assistência à saúde e saneamento são ainda muito maiores.

\section{Resumo}

Examinou-se a prevalência e fatores associados à anemia em estudo transversal com 429 crianças de 6 a 59 meses do Município de Jordão, Estado do Acre, Brasil. Modelos múltiplos de regressão de Poisson foram utilizados com seleção hierárquica das variáveis independentes. A anemia foi altamente prevalente (57,3\%; IC95\%: 52,5\%-62,1\%). Ter idade entre 6 e 23, 9 meses [razão de prevalência - RP (IC95\%): 1,40 (1,09-1,74)], morar na área rural [RP: 1,23 (1,04-1,44)], morar em domicílio com 5 a 14 crianças [RP: 1,23 (1,04-1,44)], ter mãe que fumou na gravidez [RP: 1,29 (1,09-1,53)], mãe anêmica [RP: 1,18 (1,00-1,39)] e apresentar déficit de altura para idade [RP: 1,19 (1,01-1,39)] foram fatores associados ao risco de anemia, e ter mãe que trabalha fora [RP: 0,78 (0,64-0,94)] foi fator de proteção. A anemia é um grave problema de saúde pública nesse município. Estratégias multissetoriais de combate à pobreza, aumento da cobertura e qualidade de serviços de assistência à saúde materno-infantil devem ser implementados.

Anemia; Bem-Estar da Criança; Estado Nutricional; Prevalência

\section{Colaboradores}

C. S. M. Oliveira participou da coleta, processamento, análise e interpretação dos dados, revisão da literatura e redação do artigo. M. A. Cardoso participou da organização, análise e interpretação dos dados e revisão final do artigo. T. S. Araújo contribuiu na coleta de dados, análise estatística e redação final do artigo. P. T. Muniz foi responsável pelo planejamento, concepção e obtenção de recursos para a realização do estudo, coordenou a coleta de dados e revisou a final do artigo.

\section{Agradecimentos}

À população de Jordão pela receptividade e a todos os participantes pela colaboração. À Pastoral da Criança, ao Pólo-Base de Saúde Indígena e aos entrevistadores pela inestimável contribuição durante o trabalho de campo. À Prefeitura Municipal pelo apoio logístico. A M. S. Nunes, K. K. Scopel e H. C. Koury pela participação no trabalho de campo. Ao Departamento de Ciências e Tecnologia do Ministério da Saúde pelo financiamento do estudo. 
1. Demayer E, Adiels-Tegman M. The prevalence of anaemia in the world. World Health Stat Q 1985; 38:302-17.

2. Benoist B, McLean E, Egli I, Cogswell M, editors. Worldwide prevalence of anaemia 1993-2005: WHO global database on anaemia. Geneva: World Health Organization; 2008.

3. World Health Organization/United Nations Children's Fund/United Nations University. Iron deficiency anaemia. Assessment, prevention and control. A guide for programme managers. Geneva: World Health Organization; 2001.

4. Jordão RE, Bernardi JLD, Barros Filho AA. Prevalência de anemia ferropriva no Brasil: uma revisão sistemática. Rev Paul Pediatr 2009; 27:90-8.

5. Silva SCL, Batista Filho M, Miglioli TC. Prevalência e fatores de risco de anemia em mães e filhos no Estado de Pernambuco. Rev Bras Epidemiol 2008; 11:266-77.

6. Monteiro CA, Szarfarc SC, Mondini L. Tendência secular da anemia na infância na Cidade de São Paulo (1984-1996). Rev Saúde Pública 2000; 34: 62-72.

7. Osório MM, Lira PIC, Batista-Filho M, Asworth A. Prevalence of anemia in children 6-59 months old in the state of Pernambuco, Brazil. Rev Panam Salud Pública 2001; 10:101-7.

8. Assis AMO, Barreto ML, Gomes GSS, Prado MS, Santos NS, Santos LMP, et al. Childhood anemia prevalence and associated factors in Salvador, Bahia, Brazil. Cad Saúde Pública 2004; 20:1633-41.

9. Mondini L, Cano EM, Fagundes U, Lima EES, Rodrigues D, Baruzzi RG. Condições de nutrição em crianças Kamaiurá - povo indígena do Alto Xingu, Brasil Central. Rev Bras Epidemiol 2007; 10:39-47.

10. Orellana JDY, Coimbra Jr. CEA, Lorenço AEP, Santos RV. Estado nutricional e anemia em crianças Suruí, Amazônia, Brasil. J Pediatr (Rio J.) 2006; 82:383-7.

11. Ministério da Saúde. Pesquisa Nacional de Demografia e Saúde da Criança e da Mulher - PNDS 2006: dimensões do processo reprodutivo e da saúde da criança. Brasília: Ministério da Saúde; 2009.

12. Stolztfus RJ. Iron-deficiency anemia: reexamining the nature and magnitude of the public health problem. Defining iron-deficiency anemia in public health terms: a time for reflection. J Nutr 2001; 131:565S-7.

13. Jones KM, Ramirez-Zea M, Zuleta C, Allen LH. Prevalent vitamin B-12 deficiency in twelvemonth-old Guatemalan infants is predicted by maternal B-12 deficiency and infant diet. J Nutr 2007; 137:1307-13.

14. Silva LSM, Giugliani ERJ, Aerts DRGC. Prevalência e determinantes de anemia em crianças de Porto Alegre, RS, Brasil. Rev Saúde Pública 2001; 35:6673.

15. Oliveira MAA, Osório MM, Raposo MCF. Concentração de hemoglobina e anemia em crianças no Estado de Pernambuco, Brasil: fatores sócio-econômicos e de consumo alimentar associados. Cad Saúde Pública 2006; 22:2169-78.

16. Mahoney Jr. DH. Anemia in at-risk populations what should be our focus? Am J Clin Nutr 2008; 88:1457-8.
17. Monteiro CA, Benício MHD, Konno SC, Silva ACF, Lima ALL, Conde WL. Causas do declínio da desnutrição infantil no Brasil, (1996-2007). Rev Saúde Pública 2009; 43:35-43.

18. Batista Filho M, Rissin A. Transição nutricional no Brasil: tendências regionais e temporais. Cad Saúde Pública 2003; 19 Suppl 1:S181-91.

19. Oliveira RS, Diniz AS, Benigna MJC, Miranda-Silva SM, Lola MM, Gonçalves MC, et al. Magnitude, distribuição espacial e tendência da anemia em pré-escolares da Paraíba. Rev Saúde Pública 2002; 36:26-32.

20. Fundo das Nações Unidas para a Infância. Situação da infância brasileira. Crianças de até 6 anos: o direito à sobrevivência e ao desenvolvimento. Brasília: Fundo das Nações Unidas para a Infância; 2005.

21. Benício MHD’A, Monteiro CA, Venâncio SY, Cotrin LS, Konno SC, Lima FD. Novas estimativas para a prevalência da desnutrição na infância nos 5507 municípios brasileiros a partir de modelo multinível. São Paulo: Núcleo de Pesquisas Epidemiológicas em Nutrição e Saúde, Universidade de São Paulo; 2004.

22. Agência de Notícias do Acre. Jordão: uma pequena cidade amazônica. http://www.agencia.ac.gov.br/ index.php?option=com_content\&task=view\&id=7 666\&Itemid=295 (acessado em 03/Fev/2011).

23. Instituto Brasileiro de Geografia e Estatística. Contagem da população. 2007. http://www.ibge.gov. br/home/estatistica/populacao/contagem2007/ contagem.pdf (acessado em 08/Fev/2011).

24. Filmer D, Pritchett LH. Estimating wealth effects without expenditure data-or tear: an application to educational enrolments in states of India. Demography 2001; 38:115-32.

25. World Health Organization. Physical status: the use and interpretation of anthropometry. Geneva: World Health Organization; 1995.

26. De Onis M, Onyango AW, Borghi E, Garza C, Yang H. Comparison of the World Health Organization (WHO). Child growth standards and the National Center for Health Statistics/WHO international growth reference: implications for child health programmes. Public Health Nutr 2006; 9:942-7.

27. Gleason G, Scrimschaw NS. An overview of the functional significance of iron deficiency. In: Kraemer K, Zimmermann MB editors. Nutritional anemia. Basel: Sight and Life Press; 2007. p. 58.

28. Maldonado G, Greenland S. Simulation study of confounder-selection strategies. Am J Epidemiol 1993; 138:923-36.

29. Muniz PT, Castro TG, Araújo TS, Nunes NB, SilvaNunes M, Hoffmann EEE, et al. Child health and nutrition in the Western Brazilian Amazon: population-based surveys in two counties in Acre State. Cad Saúde Pública 2007; 23:1283-93.

30. Assunção MCF, Santos IS, Barros AJD, Gigante DP, Victora CG. Anemia em menores de seis anos: estudo de base populacional em Pelotas, RS. Rev Saúde Pública 2007; 41:328-35. 
31. Howard CT, Pee S, Sari M, Bloem MW, Semba RD. Association of diarrhea with anemia among children under age five living in rural areas of Indonesia. J Trop Pediatr 2007; 53:238-44.

32. Ferreira MU, Silva-Nunes M, Bertolino CN, Malafronte RS, Muniz PT, Cardoso MA. Anemia and iron deficiency in school children, adolescents and adults: a community-based study in Rural Amazonia. Am J Public Health 2007; 97:237-9.

33. Cardoso MA, Ferreira MU, Camargo LM, Szarfarc SC. Anaemia, iron deficiency and malaria in a rural community in Brazilian Amazon. Eur J Clin Nutr 1994; 48:326-32.

34. Dang S, Yan H, Yamamoto S, Wang X, Zeng L. Feeding practice among younger Tibetan children living at high altitudes. Eur J Clin Nutr 2005; 59:1022-9.

35. Michaelsen KF, Friis H. Complementary feeding: a global perspective. Nutrition 1998; 14:763-6.

36. Neuman NA, Tanaka OU, Szarfarc SC, Guimarães PRV, Victora CG. Prevalência e fatores de risco para anemia no sul do Brasil. Rev Saúde Pública 2000; 34:56-63.

37. Matta IEA, Veiga GV, Baião MR, Santos MMAS, Luiz RR. Anemia em crianças menores de cinco anos que freqüentam creches públicas do Município do Rio de Janeiro, Brasil. Rev Bras Saúde Matern Infant 2005; 5:349-57.
38. Santos I, Cesar JA, Minten G, Valle N, Neumann NA, Cercato E. Prevalência e fatores associados à ocorrência de anemia entre menores de seis anos de idade em Pelotas, RS. Rev Bras Epidemiol 2004; 7:403-15.

39. Spinelli MGN, Marchioni DML, Souza JMP, Souza SB, Szarfarc SC. Fatores de risco para anemia em crianças de 6 a 12 meses no Brasil. Rev Panam Salud Pública 2005; 17:84-91.

40. Dallman PR, Reeves JD. Laboratory diagnosis of iron deficiency. In: Stekel A, editor. Iron nutrition in infancy and childhood. New York: Raven Press; 1984; p. 11-44.

41. Machado JB, Lopes MHI. Abordagem do tabagismo na gestação. Sci Med 2009; 19:75-80.

42. Fabian C, Olinto MTA, Costa JSD, Nácul LC. Prevalência de anemia e fatores associados em mulheres adultas residentes em São Leopoldo, Rio Grande do Sul, Brasil. Cad Saúde Pública 2007; 23: 1199-205.

43. El-Sayed N, Gad A, Nofal L, Zeid HA, El-Morshedy $\mathrm{H}$, El-Waseef S. Assessment of the prevalence and potential determinants of nutritional anaemia in Upper Egypt. Food Nutr Bull 1999; 20:417-28.

44. Miglioli TC, Brito AM, Lira PIC, Figueroa JN, Batista Filho M. Anemia no binômio mãe-filho no Estado de Pernambuco, Brasil. Cad Saúde Pública 2010; 26:1807-20.

Recebido em 08/Set/2010

Versão final reapresentada em 15/Fev/2011

Aprovado em 22/Fev/2011 